\title{
Efficient Polarization Diversity Grating Couplers in Bonded InP-Membrane
}

\author{
Frederik Van Laere, Student Member, IEEE, Tiziana Stomeo, Dirk Taillaert, Member, IEEE, \\ Günther Roelkens, Member, IEEE, Dries Van Thourhout, Member, IEEE, Thomas F. Krauss, and \\ Roel Baets, Fellow, IEEE
}

\begin{abstract}
We report on the design, fabrication, and measurement of compact and efficient grating couplers between fiber and nanophotonic waveguides, in a polarization diversity configuration. This approach allows for polarization-independent integrated circuits. The gratings and waveguides are fabricated in a benzocyclobutene (BCB)-bonded InP-membrane, with high vertical refractive index contrast. The coupling loss of the gratings is $-3.3 \mathrm{~dB}$ and the measured polarization-dependent loss is $0.79 \mathrm{~dB}$.
\end{abstract}

Index Terms-Benzocyclobutene (BCB), grating couplers, InPmembrane, polarization diversity.

\section{INTRODUCTION}

$\mathbf{H}$ IGH refractive index contrast waveguides enable large-scale integration of optical functions, since components, waveguides, and bends can be very compact. However, efficient optical coupling between an optical fiber and nanophotonic components is difficult. There are two main problems. First, there is a large mismatch in dimensions between the optical fiber mode and the waveguide mode. Simple butt-coupling results in unacceptable losses. Second, there is a polarization problem. Light from the fiber has an unknown polarization, which changes over time. However, the on-chip optical components are often very polarization-sensitive, especially when using high refractive index contrast, so, even when the fiber-to-chip coupling can be made polarization-independent, the actual nanophotonic components cannot, therefore requiring on-chip polarization splitters and rotators [1], [2]. Efficient edge-coupling methods between fiber and high index contrast waveguides have been demonstrated [3]-[5]. However, they do not solve these polarization issues.

Compact grating couplers for out-of plane coupling between standard single-mode optical fiber and high index contrast waveguides are reviewed in [6]. As no polished or cleaved facet is required, they allow for wafer-scale testing. While 1-D

Manuscript received July 31, 2007; revised October 24, 2007. This work was supported by the the EU through the FP6-IST-004582 FUNFOX Project. The work of F. Van Laere and D. Taillaert was supported by the Institute for the Promotion of Innovation through Science and Technology (IWT Flanders) under a scholarship and a postdoctoral grant, respectively. The work of G. Roelkens was supported by the Fund for Scientific Research (FWO Flanders) under a postdoctoral grant.

F. Van Laere, D. Taillaert, G. Roelkens, D. Van Thourhout, and R. Baets are with the Department of Information Technology, Ghent University-IMEC, B-9000 Gent, Belgium (e-mail: frederik.vanlaere@ intec.ugent.be).

T. Stomeo and T. F. Krauss are with the School of Physics and Astronomy, University of St. Andrews, St. Andrews, Fife KY16 9SS, U.K.

Color versions of one or more of the figures in this letter are available online at http://ieeexplore.ieee.org.

Digital Object Identifier 10.1109/LPT.2007.915587 gratings are very polarization selective, a 2-D grating can be used in a polarization diversity configuration, without the need for integrated polarization splitters and rotators. A polarization diversity duplexer was demonstrated in silicon-on-insulator, with a polarization-dependent loss (PDL) of $0.66 \mathrm{~dB}$ and a coupling loss of $-6.7 \mathrm{~dB}$ [7].

Due to its indirect bandgap, it is very difficult to make active devices in silicon. However, III-V materials (and more specifically InP-InGaAsP for telecom applications) are very well suited for this purpose. While only a small vertical refractive index contrast can be achieved in a classical InP-heterostructure, a high omnidirectional contrast can be achieved using wafer bonding [8] or underetching. The high vertical index contrast can then be used for components requiring this high contrast, which can still be integrated with classical InP-based layer structures for active devices.

Here, we report on polarization diversity grating couplers in InP membrane. The final device is an efficient and polarization-independent optical link from fiber-to-fiber. The efficiency of the grating couplers substantially improved to $47 \%$ (coupling loss of $-3.3 \mathrm{~dB}$ ) due to the incorporation of a bottom gold mirror [9] and the PDL is $0.79 \mathrm{~dB}$.

\section{PolariZATION Diversity Using 2-D GRATINGS}

\section{A. Principle}

In a polarization diversity approach, the two orthogonal polarization components in the fiber are typically split and one polarization component is rotated $90^{\circ}$. Both, now equal polarizations, are then guided towards two identical nanophotonic (polarization-sensitive) circuits. At the output, the reverse operation is performed and the polarization components (orthogonal again) are recombined.

We use a 2-D diffractive grating for performing fiber coupling and (integrated) polarization diversity in one device, as shown in Figs. 1 and 2 and described in [10]. The structure consists of a 2-D grating at the intersection of two orthogonal waveguides. For symmetry reasons, the two orthogonal polarization components coming from a vertical fiber couple to their own waveguide, in which the polarization is the same.

\section{B. Near Vertical Coupling}

Vertical coupling suffers from large second-order reflections. This can be circumvented by tilting the fiber along the bisection line of the waveguides (for symmetry reasons), which is shown in Fig. 2 (left). However, this tilt of the fiber reduces coupling to the fundamental waveguide mode of orthogonally positioned waveguides, due to a mismatch in the phase front of the optical waveguide mode and that of the diffracted light. This 


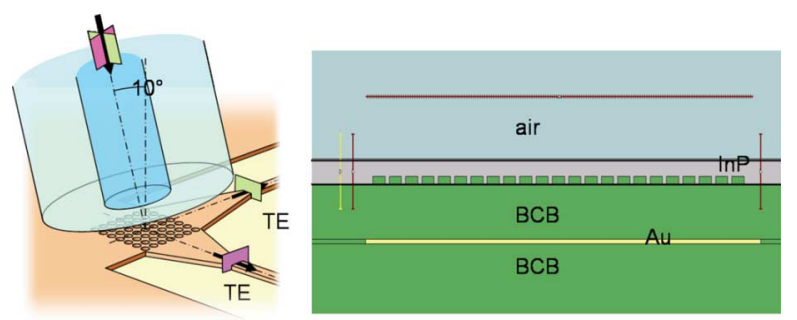

Fig. 1. (Left) Polarization diversity using 2-D grating couplers and a (near) vertical positioned fiber. (Right) Cross section of the simulated structure.
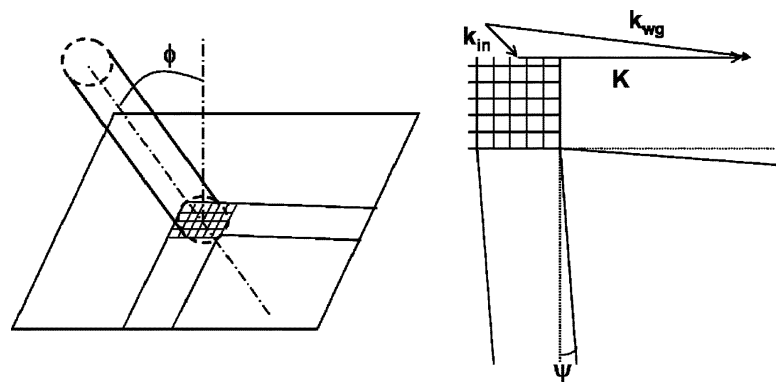

Fig. 2. Top view. (Left) 2-D grating with near vertical coupling and rotated waveguides. (Right) $K$-vector diagram. Angles $\phi$ and $\psi$ are indicated.

can be solved by rotating the waveguides (while preserving the symmetry along the bisection line). We explain this effect by a $k$-vector diagram [Fig. 2 (right)]. The relation

$$
\vec{k}_{\mathrm{wg}}=\vec{k}_{\text {in,proj }}+\vec{K}
$$

with $\vec{k}_{\text {wg }}$ the wave vector of the optical waveguide mode, $\vec{k}_{\text {in,proj }}$, the projected wave vector of the incident fiber mode, and $\vec{K}$ the reciprocal lattice vector of the grating, results in the following equations:

$$
\begin{aligned}
& \frac{2 \pi}{\lambda} n_{\text {eff }} \sin (\psi)=\frac{2 \pi}{\lambda} \sin (\phi) \cos \left(\frac{\pi}{4}\right) \\
& \frac{2 \pi}{\lambda} n_{\text {eff }} \cos (\psi)=\frac{2 \pi}{\lambda} \sin (\phi) \cos \left(\frac{\pi}{4}\right)+\frac{2 \pi}{\Lambda}
\end{aligned}
$$

with $\phi$ the tilt angle of the optical fiber [Fig. 2 (left)] and $\psi$ the angle of the waveguide [Fig. 2 (right)]. For a given angle $\phi$ of the fiber, (2) can be used to calculate the waveguide angle $\psi$. And for these values $\phi$ and $\psi$, (3) can be used to calculate the grating period. The effective index $n_{\text {eff }}$ is the effective index of the waveguide mode in the case of a weak grating. For high index contrast gratings, this is an approximation and rigorous design requires full 3-D calculations on the entire structure (gratings and rotated waveguides together). We have not done these simulations, but we have experimentally verified these effects.

\section{DESIGN}

The 2-D grating parameters were obtained using 3-D finitedifference time-domain (FDTD) simulations (grid of $20 \mathrm{~nm}$ ) on a reduced structure. In order to keep the computational effort within limits, only one row of holes ( 20 periods) in the lateral direction was simulated. A cross section of the structure is shown in Fig. 1 (right). The optical power that is diffracted upwards was modeled. As no light is diffracted downwards due to the

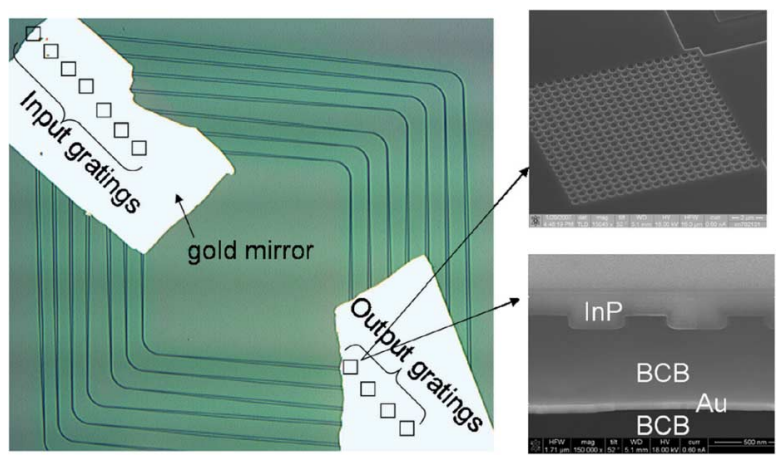

Fig. 3. (Left) Microscope image of the sample before bonding. (Light-hatched regions are gold covering the gratings. They will form the bottom mirror after bonding.) Gratings are inside the rectangles. (Top right) Top scanning electron microscope view of the grating before bonding. (Bottom right) Cross section of a fabricated structure after bonding.

presence of the gold mirror, the coupling strength of the grating and from that the coupling to a Gaussian fiber mode can be calculated [6], [11]. This results in a period of $640 \mathrm{~nm}$ (near vertical coupling at $10^{\circ}$ ), an etch depth of $100 \mathrm{~nm}$, and a hole diameter of $420 \mathrm{~nm}$. The benzocyclobutene (BCB) spacing layer between the InP-membrane grating and the gold bottom mirror has a major influence on the coupling efficieny [9], [12]. Coupling is optimal for a $620-\mathrm{nm}$ BCB spacer layer. For $\phi=10^{\circ}$ and $n_{\mathrm{eff}}=2.73$ ( $n_{\mathrm{eff}}$ of the waveguide), (2) results in $\psi=2.57^{\circ}$. Using these values, (3) results in a period of $595 \mathrm{~nm}$ (instead of the simulated $640 \mathrm{~nm}$ ), the discrepancy being due the fact that (3) assumes weak gratings, which is not the case here.

\section{FABRICATION}

The layer structure consists of a 300-nm InP-membrane layer on top of a InGaAsP $\left(\lambda_{g}=1.22 \mu \mathrm{m}\right)$ etch-stop layer on an InP-substrate. Gratings $(12 \mu \mathrm{m} \times 12 \mu \mathrm{m})$ and waveguides were defined with e-beam lithography and etched into the InP-membrane layer to a depth of $100 \mathrm{~nm}$. Afterwards, a BCB layer of $620 \mathrm{~nm}$ was spin-coated onto the InP-die, and cured for $1 \mathrm{~h}$ at $250{ }^{\circ} \mathrm{C}$ in a nitrogen environment. A 60 -nm-thick gold layer was evaporated above the gratings, using lift-off. This gold layer will serve as the grating bottom mirror. Then, the InP-sample was bonded onto a GaAs host-substrate with a $1-\mu \mathrm{m}$-thick BCBlayer [8]. This BCB-layer was also cured for $1 \mathrm{~h}$ at $250{ }^{\circ} \mathrm{C}$ in a nitrogen environment. The InP-substrate was removed using lapping and wet-etching in $\mathrm{HCl}$ until the InGaAsP etch stop layer was reached. Finally, the $\mathrm{InGaAsP}$ etch-stop layer was removed using wet-etching in $\mathrm{H}_{2} \mathrm{O}: \mathrm{H}_{2} \mathrm{SO}_{4}: \mathrm{H}_{2} \mathrm{O}_{2}$. A picture of the structure prior to bonding and a cross section is shown in Fig. 3.

\section{Measurements}

\section{A. Determination of the Optimal Waveguide Angle}

First, the influence of the waveguide angle was assessed. The layout of the structure is shown in Fig. 3. Both input and output grating are placed at the intersection of two $(12 \mu \mathrm{m}$ wide $)$ nearly orthogonal waveguides. The wide waveguides are tapered adiabatically to single-mode bent waveguides (600 $\mathrm{nm}$ wide), in order to connect input and output gratings. On the sample, the waveguide angles are varied. A standard SMF-28 input fiber, connected with a tunable laser is positioned above the input 


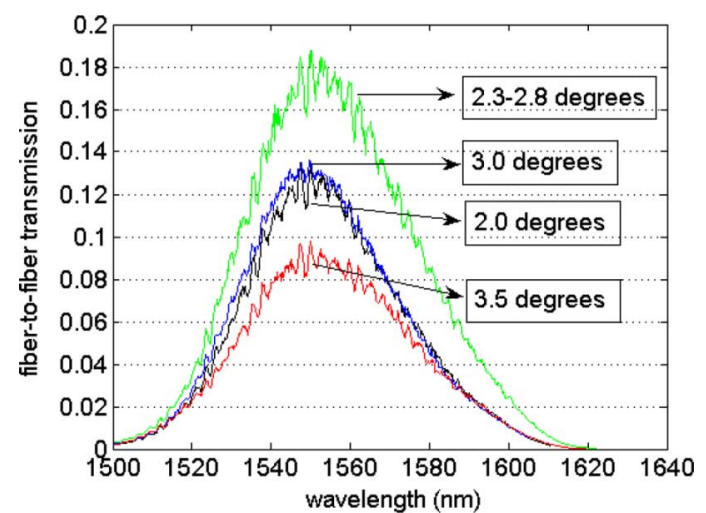

Fig. 4. Measured spectra for structures with different waveguide angles (for clarity, overlapping curves are not shown).

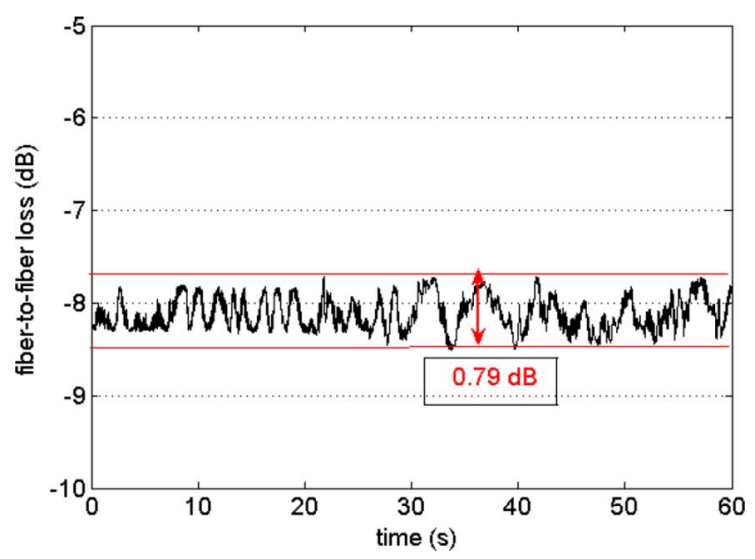

Fig. 5. Measurement of PDL.

grating. The input light for these measurements is linearly polarized, with the electric field along the diagonal of the 2-D grating. For this polarization, the coupling efficiency to both waveguides is the same. An output fiber is positioned over the output grating. The fiber positions are optimized for maximum transmission. For waveguide angles varying from $2.3^{\circ}$ to $2.8^{\circ}$, the fiber-to-fiber loss is $7.3 \mathrm{~dB}$. Subtracting the loss in the tapers and bends $(1 \mathrm{~dB})$ determined from reference structures on the sample, results in a loss of $3.15 \mathrm{~dB}$ per coupler or an efficiency of $48 \%$. The $1-\mathrm{dB}$ bandwidth is $40 \mathrm{~nm}$. For the other structures, the waveguide angle is not optimal, and there is less coupling to the fundamental mode of the waveguide (which is transmitted through the single-mode bend). The measurements are shown in Fig. 4.

\section{B. Determination of PDL}

In theory, the above method for aligning the fibers yields also the optimal low PDL. However, in practice, no perfect symmetry (along the bisection line of the gratings) can be obtained, and the optimal fiber positions for minimum PDL deviates slightly from the former optimal fiber position. The fiber positions were, therefore, optimized in order to minimize the PDL. This PDL is measured by changing the input polarization randomly over time using polarization control paddles and measuring the vari- ation in output power. This results in a slight additional loss of $0.15 \mathrm{~dB}$ per coupler (grating coupler efficiency of $47 \%$ ). The minimum measured PDL at the center wavelength of the grating coupler spectrum is $0.79 \mathrm{~dB}$, as shown in Fig. 5. This PDL is caused by slight asymmetry in the structure (gratings, waveguide arms), due to fabrication imperfections. Coupling and propagation conditions are then slightly different for both orthogonal polarization components of the input light. Also, phase differences at the output coupler play a role when using nonorthogonal waveguides.

\section{CONCLUSION}

We have reported on efficient polarization diversity grating couplers in BCB-bonded InP-membranes. The fiber-to-fiber loss is $-7.6 \mathrm{~dB}$, the grating coupler loss is $-3.3 \mathrm{~dB}(47 \%$ efficiency), and the PDL is $0.79 \mathrm{~dB}$.

\section{ACKNOWLEDGMENT}

The Fraunhofer Institut fur Nachrichtentechnik, Heinrich Hertz Institut, Berlin, is acknowledged for providing the InP epiwafers.

\section{REFERENCES}

[1] H. Fukuda, K. Yamada, T. Tsuchizawa, T. Watanabe, H. Shinojima, and S. Itabashi, "Ultrasmall polarization splitter based on silicon wire waveguides," Opt. Express, vol. 14, pp. 12401-12408, 2006.

[2] T. Barwicz, M. R. Watts, M. Popovic, P. T. Rakich, L. Socci, F. X. Kartner, E. P. Ippen, and H. I. Smith, "Polarization-transparent microphotonic devices in the strong confinement limit," Nature Photon., vol. 1 , pp. 57-60, 2007.

[3] T. Shoji, T. Tsuchizawa, T. Watanabe, K. Yamada, and H. Morita, "Low loss mode size converter from $0.3 \mu \mathrm{m}$ square $\mathrm{Si}$ wire waveguides to singlemode fibres," Electron. Lett., vol. 38, pp. 1669-1670, 2002.

[4] V. R. Almeida, R. R. Panepucci, and M. Lipson, "Nanotaper for compact mode conversion," Opt. Lett., vol. 28, pp. 1302-1304, Aug. 2003.

[5] P. Cheben, D. X. Xu, S. Janz, and A. Densmore, "Subwavelength waveguide grating for mode conversion and light coupling in integrated optics," Opt. Express, vol. 14, pp. 4695-4702, May 2006.

[6] D. Taillaert, F. Van Laere, M. Ayre, W. Bogaerts, D. Van Thourhout, P. Bienstman, and R. Baets, "Grating couplers for coupling between optical fibers and nanophotonic waveguides," Jpn. J. Appl. Phys. Part 1-Regular Papers Brief Commun. Rev. Papers, vol. 45, pp. 6071-6077, Aug. 2006.

[7] W. Bogaerts, D. Taillaert, P. Dumon, D. Van Thourhout, and R. Baets, "A polarization-diversity wavelength duplexer circuit in silicon-on-insulator photonic wires," Opt. Express, vol. 15, pp. 1567-1578, Feb. 2007.

[8] G. Roelkens, J. Brouckaert, D. Van Thourhout, R. Baets, R. Nötzel, and M. Smit, "Adhesive bonding of InP/InGaAsP dies to processed silicon-on-insulator wafers using DVS-bis-Benzocyclobutene," J. Electrochemical Soc., vol. 153, pp. G1015-G1019.

[9] F. Van Laere, G. Roelkens, M. Ayre, J. Schrauwen, D. Taillaert, D. Van Thourhout, T. F. Krauss, and R. Baets, "Compact and highly efficient grating couplers between optical fiber and nanophotonic waveguides," J. Lightw. Technol., vol. 25, no. 1, pp. 151-156, Jan. 2007.

[10] D. Taillaert, H. Chong, P. Borel, L. Frandsen, R. M. De La Rue, and R. Baets, "A compact two-dimensional grating coupler used as a polarization splitter," IEEE Photon. Technol. Lett., vol. 15, no. 9, pp. 1249-1251, Sep. 2003.

[11] R. Orobtchouk, A. Layadi, H. Gualous, D. Pascal, A. Koster, and S. Laval, "High efficiency light coupling in a submicrometric silicon-oninsulator waveguide," Appl. Opt., vol. 39, pp. 5773-5777, 2000.

[12] R. M. Emmons and D. G. Hall, "Buried-oxide silicon-on-insulator structures II: Waveguide grating couplers," IEEE J. Quantum Electron., vol. 28, no. 1, pp. 164-175, Jan. 1992. 